\title{
Icariin protects against ischemia-reperfusion injury in H9C2 cells by upregulating heat shock protein 20
}

\author{
ZHI-HONG REN ${ }^{1 *}$, ZUN-PING KE ${ }^{2 *}$, MAN LUO $^{3}$ and YAN SHI \\ ${ }^{1}$ Department of Pediatrics, The Central Hospital of Huanggang, Huanggang, Hubei 438000; \\ ${ }^{2}$ Department of Cardiology, The Fifth People's Hospital of Shanghai, Fudan University, Shanghai 200240; \\ ${ }^{3}$ Department of Emergency, Huai'an First People's Hospital, Nanjing Medical University, Huai'an, Jiangsu 223300; \\ ${ }^{4}$ Department of Emergency, The Affiliated Huai'an Hospital of Xuzhou Medical University and \\ The Second People's Hospital of Huai'an, Huai'an, Jiangsu 223302, P.R. China
}

Received October 28, 2016; Accepted June 30, 2017

DOI: $10.3892 / \mathrm{mmr} .2017 .8251$

\begin{abstract}
Icariin (ICA) has been implicated in certain biological and pathological processes, including myocardial ischemia/reperfusion (I/R) injury. The aim of the present study was to investigate the role of ICA in I/R-induced cardiomyocyte injury and the potential underlying mechanism. Cell proliferation and apoptosis of $\mathrm{H} 9 \mathrm{C} 2$ cells was determined by cell counting kit- 8 and flow cytometry assays. In addition, reactive oxygen species (ROS) production in H9C2 cells was measured by flow cytometry. Reverse transcription-quantitative polymerase chain reaction and western blot assay were performed to examine the expression levels of proteins, including HSP20, B-cell lymphoma 2 (Bcl-2), cytochrome complex (Cyt-c), apoptotic protease activating factor 1 (APAF1), caspase-9 andcaspase-3, and the phosphorylation of Akt (p-Akt) in $\mathrm{H} 9 \mathrm{C} 2$ cells. The present results demonstrated that, compared with the control group, the I/R group demonstrated significantly reduced levels of HSP20 expression and cell proliferation, and increased apoptosis and ROS production in $\mathrm{H} 9 \mathrm{C} 2$ cells. In parallel, the expression levels of Cyt-c, APAF1, caspase-9 and caspase- 3 were significantly increased in the I/R group, although Bcl-2 and p-Akt/Akt expression levels were decreased. Furthermore, compared with the I/R group, ICA
\end{abstract}

Correspondence to: Dr Man Luo, Department of Emergency, Huai'an First People's Hospital, Nanjing Medical University, 6 West Beijing Road, Huai'an, Jiangsu 223300, P.R. China

E-mail: manluol@163.com

Dr Yan Shi, Department of Emergency, The Affiliated Huai'an Hospital of Xuzhou Medical University and The Second People's Hospital of Huai'an, 62 South Huai'hai Road, Huai'an, Jiangsu 223302, P.R. China

E-mail: jfmsy@163.com

${ }^{*}$ Contributed equally

Key words: myocardial ischemia/reperfusion, icariin, heat shock protein 20, apoptosis treatment and/or HSP20 overexpression significantly improved cardiac function, as evidenced by promoted cell proliferation and inhibited apoptosis of H9C2 cells. The current study indicates that ICA exerts a cardioprotective effect against I/R injury, which is associated with the upregulation of HSP20.

\section{Introduction}

Myocardial ischemia refers to the heart with blood perfusion reduction, which occurs when the balance of myocardial blood supply and demand is disturbed (1), resulting in abnormal metabolism of oxygen and energy, and the abnormal pathological state of the heart (2). The definition of myocardial ischemia/reperfusion (I/R) injury is that the ischemic myocardium restores blood flow following reperfusion, which affects the prognosis of patients with myocardial infarction (3). This is followed by the disordered synthesis of mitochondrial energy and $\mathrm{Ca}^{2+}$ homeostasis, release of free radicals and inflammatory cytokines, and eventually leads to myocardial cell apoptosis and organ damage (4,5). Myocardial I/R injury, which represents a major cause of morbidity and mortality in humans with coronary heart disease, has complex molecular mechanisms $(6,7)$. Furthermore, the molecular mechanisms that regulates gene expression during myocardial I/R are not completely understood.

Icariin (ICA) is a flavonoid extracted from Epimedium brevicornum, a genus of flowering plants in the family, Berberidaceae (8), which is used in Traditional Chinese Herbal Medicine, and possesses multiple pharmaceutical and biological activities, such as immunoregulation (9), antioxidation (10), anti-tumor activity (11), neuroprotection (12) and improves sexual function (8). Icariin attenuates cerebral I/R injury via inhibition of inflammatory responses mediated by nuclear factor $(\mathrm{NF})-\kappa \mathrm{B}$, peroxisome proliferator-activated receptor (PPAR) $\alpha$ and PPAR $\gamma$ in rats (13). Furthermore, post-conditioning with icariin exerts cardioprotective effects against myocardial I/R injury by activating the phosphoinositide 3-kinase (PI3K)/Akt signaling pathway $(14,15)$. Given the cardioprotective role and anti-I/R effect of ICA, the current study hypothesized that ICA may present as a novel therapeutic agent for the treatment of myocardial I/R. 
Increasing evidences supports a pivotal role for the small heat shock protein (HSP) family in multiple processes (16), including tumorigenesis (17), cardioprotection (18), resistant to oxidative stress (19) and apoptosis (20). HSP20 is the best characterized small HSP compared with other small HSPs and is predominantly upregulated in animal hearts with ischemic conditions (21). Previous studies demonstrated that HSP20 protected hearts against cardiac myocyte apoptosis, induced by $\mathrm{I} / \mathrm{R}$ injury in vivo and in vivo $(22,23)$. Knockdown of endogenous miR-320 provided protection against I/R-induced cardiomyocyte apoptosis by targeting HSP20 (24). However, the potential benefits of HSP20 action on ICA-induced cardiac protection and its underlying mechanism(s) remain largely unknown.

The present study was designed to further determine the cardioprotective effect of ICA on myocardial I/R injury and the molecular mechanism underlying HSP20.

\section{Materials and methods}

Cell culture. H9C2 cells (Institute of Biochemistry and Cell Biology, Shanghai, China) were cultured in Dulbecco's modified Eagle's medium (DMEM) containing 10\% fetal calf serum (Invitrogen; Thermo Fisher Scientific, Inc., Waltham, MA, USA) and 1\% 100X mycillin (Invitrogen; Thermo Fisher Scientific, Inc.), and incubated in $5 \% \mathrm{CO}_{2}$ at $37^{\circ} \mathrm{C}$ overnight. Cells were digested and seeded into 96 -well plates $\left(3 \times 10^{3}\right.$ cells/well). The I/R group was transferred into sugar and serum-free DMEM and incubated in $5 \% \mathrm{CO}_{2}$ and $95 \% \mathrm{~N}_{2}$ at $37^{\circ} \mathrm{C}$ for $2 \mathrm{~h}$, then transferred into normal DMEM and incubated in $5 \% \mathrm{CO}_{2}$ at $37^{\circ} \mathrm{C}$ for $6 \mathrm{~h}$. The control group was incubated in normal DMEM (Invitrogen; Thermo Fisher Scientific, Inc.) for $8 \mathrm{~h}$ at $37^{\circ} \mathrm{C}$. In the drug-treated groups, ICA (Xian Xiao Cao Botanical Development Co., Ltd., Xian, China) at various concentrations $(5,10$ and $15 \mu \mathrm{mol} / \mathrm{l})$ was administered as a component of the perfusion medium $10 \mathrm{~min}$ before ischemia and every $8 \mathrm{~h}$ throughout reperfusion.

Construction of recombinant adenoviruses. The HSP20 coding sequence (commercially unavailable; Sangon Biotech Co., Ltd., Shanghai, China) was cloned into the pAVsi 1.1 adenovirus vector and black pAVsi 1.1 adenovirus vector (both Sangon Biotech Co., Ltd.) served as a negative control. To generate a high-titer adenovirus, vectors encoding the HSP20 and the packaging plasmids were cotransfected into 293T cells (Institute of Biochemistry and Cell Biology) using Lipofectamine 2000 (Invitrogen; Thermo Fisher Scientific, Inc.) according to the manufacturer's instructions. The packaging, purification and titration of adenovirus were performed as previously described (25). Following transfection (at $48 \mathrm{~h}$ ), the cell culture medium containing viral particles was collected via centrifugation at $1,000 \mathrm{x}$ g for $5 \mathrm{~min}$ at $4^{\circ} \mathrm{C}$.

Cell proliferation assay. H9C2 cells ( $1 \times 10^{3}$ cells/well) were plated in 96-well plates. Following ICA treatment for $48 \mathrm{~h}$, 10\% Cell Counting-kit 8 (CCK-8; CK04; Dojindo Molecular Technologies, Inc., Kumamoto, Japan) diluted in DMEM was mixed in each well for another $1 \mathrm{~h}$. The absorption of each sample was measured at a wavelength of $450 \mathrm{~nm}$ using a Labsystems MK3 microplate reader (Thermo Fisher Scientific,
Inc.) to detect cell viability according to the manufacturer's instruction.

Cell apoptosis assay. Following transfection, H9C2 cells were detached using $0.25 \%$ trypsin and washed with $10 \%$ phosphate-buffered saline (PBS), followed by the centrifugation at $1,000 \mathrm{x} \mathrm{g}$ for $5 \mathrm{~min}$ at $37^{\circ} \mathrm{C}$. Then, the cells were incubated with $10 \mu \mathrm{l}$ Annexin V-fluorescein isothiocyanate (FITC) and $5 \mu \mathrm{l}$ propidium iodide $(\mathrm{PI})$ in the dark for $15 \mathrm{~min}$ at $4^{\circ} \mathrm{C}$. Cell apoptotic rate was measured by Annexin V-FITC Apoptosis Detection kit (Beyotime Biotechnology, Shanghai, China) and the data was obtained using flow cytometer (BD Accuri C6 software version 1.0.264.21; BD Biosciences, Franklin Lakes, NJ, USA).

Intracellular reactive oxygen species (ROS) assay. The intracellular ROS content was determined using a fluorescent probe, 2',7'dichlorodihydrofluorescein-diacetate (DCFH-DA) followed by flow cytometry. After transfection, H9C2 cells were incubated with $10 \mu \mathrm{M}$ DCFH-DA at $37^{\circ} \mathrm{C}$ for $20 \mathrm{~min}$ in the dark. Then, the plates were washed three times with PBS. The fluorescent probe DCFH-DA (Thermo Fisher Scientific, Inc.), which detected ROS production, was observed using a flow cytometer (BD Accuri C6 software version 1.0.264.21; BD Biosciences).

$m R N A$ quantification by reverse transcription-quantitative polymerase chain reaction $(R T-q P C R)$. Total RNA was isolated from H9C2 cells using the TRIzol (Invitrogen; Thermo Fisher Scientific, Inc.) method, depurated with an RNAeasy kit (Invitrogen; Thermo Fisher Scientific, Inc.) and reversed to cDNA using the Prime-Script RT reagent kit (Takara Bio, Inc., Otsu, Japan). qPCR was performed using an ABI 7500 (Applied Biosystems; Thermo Fisher Scientific, Inc.) using SYBR Premix Ex Taq (Takara Bio, Inc.). The following primers were used: Sense, 5'-CTGTTTTGGTGAGGGGAA GG-3' and antisense, 5'-CTGGGGAGAAATGGGATACG-3' for HSP20; sense, 5'-GTCGGTGTGAACGGATTTG-3' and antisense, 5'-TCCCATTCTCAGCCTTGAC-3' for GAPDH. The HSP20 mRNA level was normalized against internal GAPDH mRNA. The relative quantification values for gene expression levels were calculated using $2^{-\Delta \Delta \mathrm{Cq}}$ method (26).

Western blot analysis. Upon termination of treatment, H9C2 cells were harvested and resuspended in ice-cold cell lysis solution and the homogenate was centrifuged at $400 \mathrm{x}$ g for $15 \mathrm{~min}$ at $4^{\circ} \mathrm{C}$. A bicinchoninic acid protein quantification kit (cat. no. 23225; Thermo Fisher Scientific, Inc.) was used to quantify the protein contents. Then, $30 \mu \mathrm{g}$ protein was run on $12 \%$ SDS-PAGE gel and transferred to a nitrocellulose filter membrane (Merck KGaA, Darmstadt, Germany) electrophoretically. Blots were blocked with 5\% skimmed milk at room temperature for $1 \mathrm{~h}$, followed by incubation with anti-B-cell lymphoma 2 (Bcl-2; cat. no. sc-492; 1:150; Santa Cruz Biotechnology, Inc., Dallas, TX, USA), caspase-3 (cat. no. ab2302; 1:200; Abcam, Cambridge, MA, USA), caspase-9 (cat. no. ab2013; 1:1,000; Abcam), cytochrome complex (Cyt-c; cat. no. ab13575; 1:1,000; Abcam), apoptotic protease activating factor 1 (APAF1; cat. no. 8969; 1:1,000; Cell Signaling Technology, Inc., Danvers, MA, USA), Akt (cat. no. 2920; 1:2,000; Cell Signaling Technology, Inc.), 
A

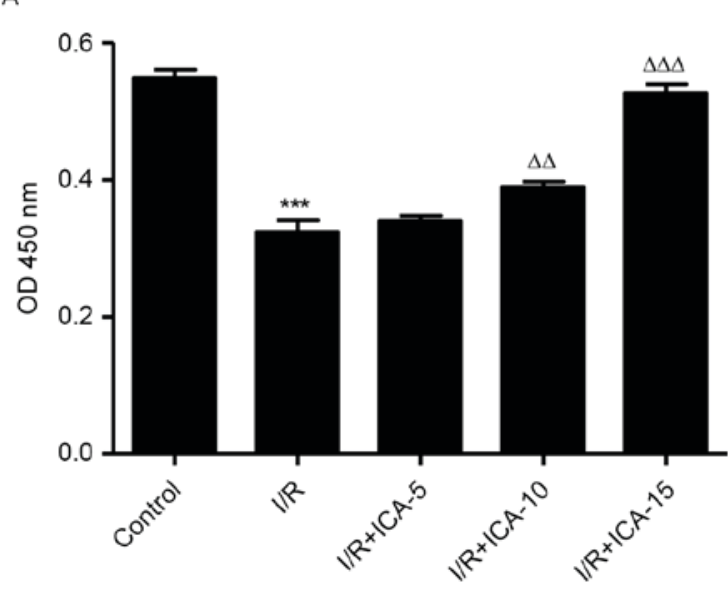

B

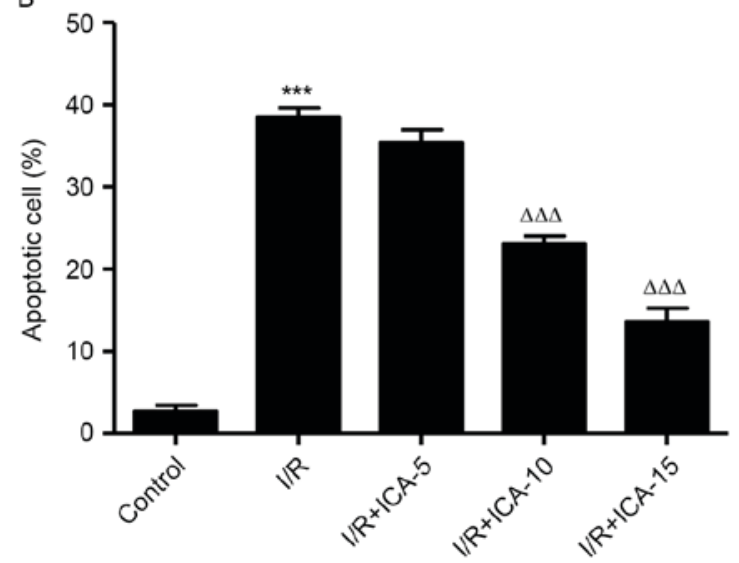

C
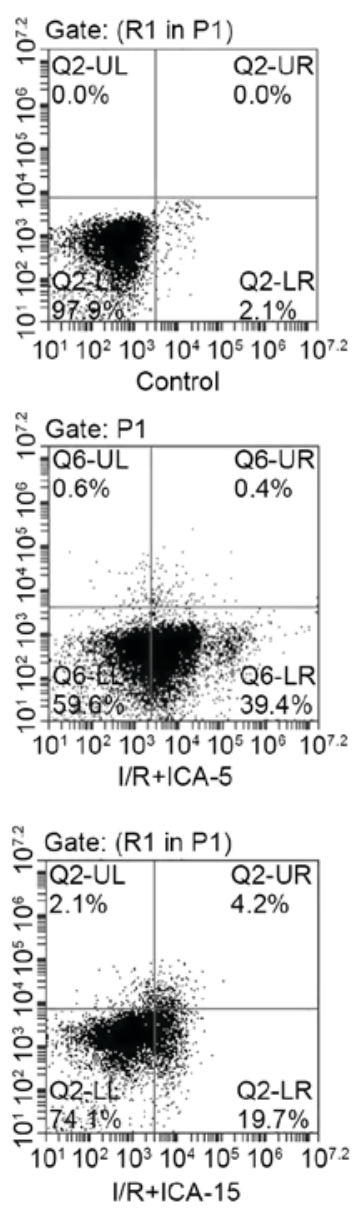
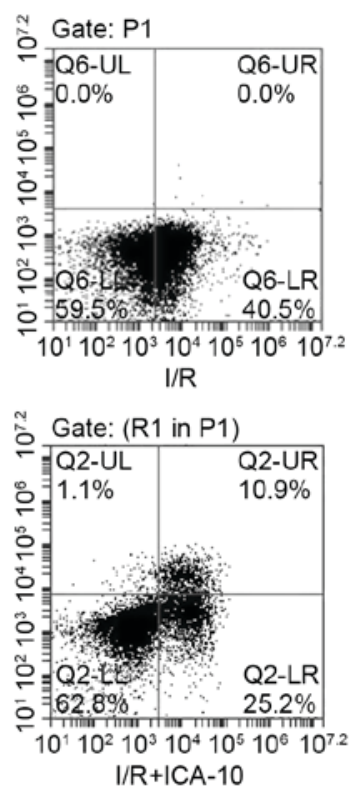

$1 / R+\mid C A-10$

Figure 1. Effects of ICA on I/R-induced injury in H9C2 cells. (A) Cell proliferation and (B and C) apoptosis in H9C2 cells following I/R treatment in the absence and presence of ICA treatment were evaluated by Cell Counting Kit- 8 assay and flow cytometry, respectively. Data are presented as the mean \pm standard deviation. ${ }^{* * *} \mathrm{P}<0.001$ vs. control. ${ }^{\Delta \Delta} \mathrm{P}<0.01,{ }^{\Delta \Delta \Delta} \mathrm{P}<0.001$ vs. I/R. ICA, icariin; I/R, ischemia/reperfusion; OD, optical density.

p-AKT (cat. no. 4060; 1:2,000; Cell Signaling Technology, Inc.) and GAPDH (cat. no. 5174; 1:1,500; Cell Signaling Technology, Inc.) antibodies overnight at $4^{\circ} \mathrm{C}$, and incubated with horse radish peroxidase-conjugated goat anti-mouse or anti-rabbit secondary antibody (cat nos. A0208 and A0216, respectively; 1:1,000; Beyotime Institute of Biotechnology, Shanghai, China) for $1 \mathrm{~h}$ at $37^{\circ} \mathrm{C}$. Enhanced chemiluminescence (Thermo Scientific, Shanghai, China) was used to detect the blots visually and signals were quantified by densitometry (Quantity One software version 4.62; Bio-Rad Laboratories, Inc., Hercules, CA, USA).

Statistical analysis. Experiments were performed in triplicate and data were expressed as the mean \pm standard deviation of the mean. Statistical significance was determined by unpaired two-tailed t-test and one-way analysis of variance followed by Tukey's post hoc test. Statistical analyses were performed using GraphPad Prism 5 software (GraphPad Software, Inc., La Jolla, CA, USA) and $\mathrm{P}<0.05$ was considered to indicate a statistically significant difference.

\section{Results}

ICA improves cell viability and inhibits cell apoptosis in H9C2 cells. In order to investigate the possible mechanisms involved in the protective effect of ICA on cardiac cells against I/R injury, an in vitro study was performed using H9C2 cells. CCK-8 assay was performed to evaluate the cell viability of H9C2 cells following I/R. Compared with the I/R group, cell proliferation in the ICA (10 and $15 \mu \mathrm{mol} / \mathrm{l})$-treated groups increased in a dose-dependent manner (Fig. 1A), indicating the improved cell viability of H9C2 cells following treatment with ICA. Cell apoptosis induced by I/R was visually detected using Annexin-V FITC/PI double staining. It was demonstrated that the apoptotic cells of ICA (10 and $15 \mu \mathrm{mol} / \mathrm{l})$-treated H9C2 cells reduced in a dose-dependent manner when compared with that of the I/R group (Fig. 1B and C), revealing the inhibitory effect of ICA against I/R-induced apoptosis.

Effect of ICA on HSP20 expression levels. To clarify the effect of I/R on HSP20 expression levels in vitro, RT-qPCR and western blot analysis were performed. As shown in Fig. 2A, the mRNA expression level of HSP20 was significantly decreased in the I/R model. Furthermore, the mRNA expression level of HSP20 was dose-dependently increased by ICA treatment (5, 10 and $15 \mu \mathrm{mol} / \mathrm{l})$. Similarly, the protein expression levels of HSP20 were also decreased following I/R, in which ICA dose-dependently increased HSP20 levels were observed in the I/R induced H9C2 cells (Fig. 2B). HSP20 overexpression significantly increased the expression level of HSP20 in 

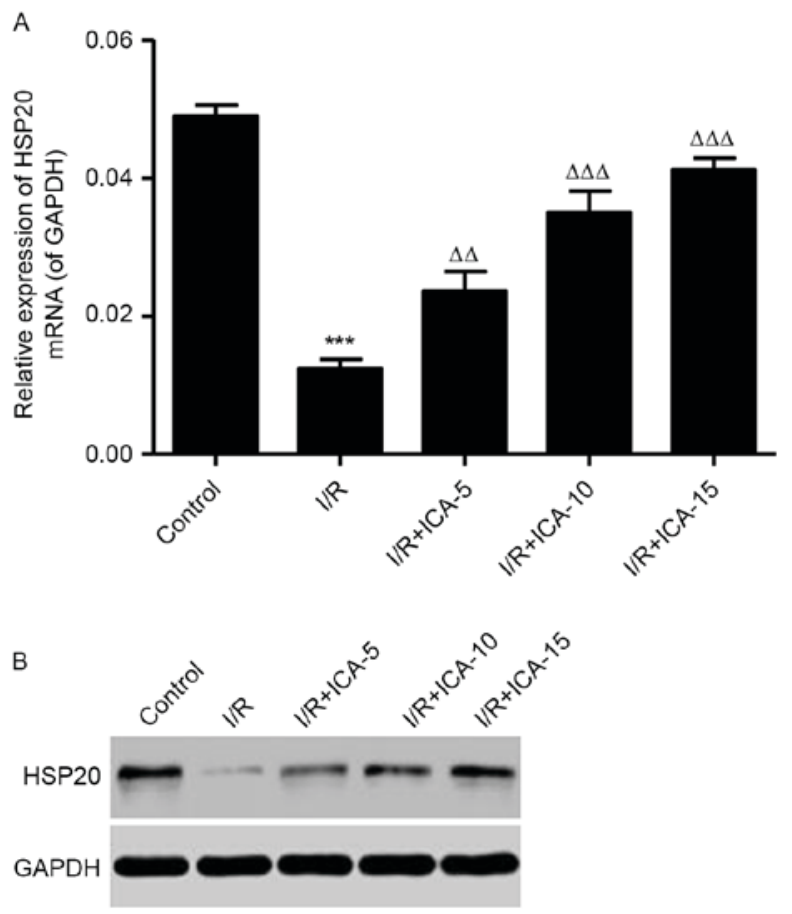

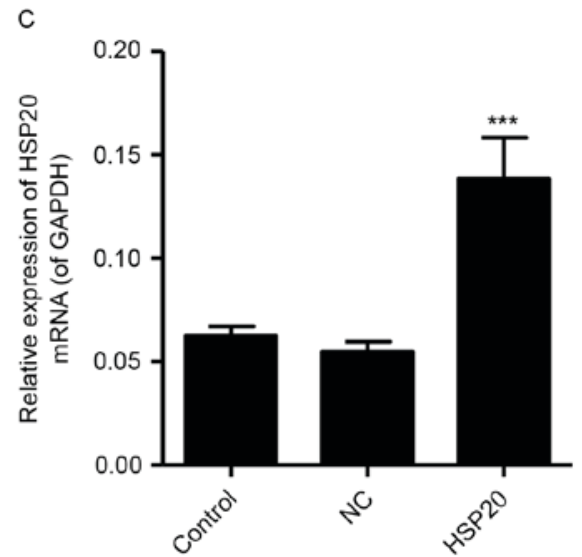

D

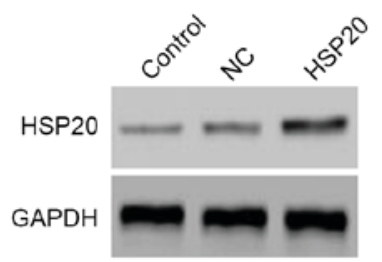

Figure 2. Expression levels of HSP20 in H9C2 cells following I/R treatment in the absence and presence of ICA treatment by (A) RT-qPCR and (B) western blot analysis. Expression levels of HSP20 in H9C2 cells following recombinant adenovirus transfection for $48 \mathrm{~h}$ by (C) RT-qPCR and (D) western blot analysis. Data are presented as the mean \pm standard deviation. ${ }^{* * * *} \mathrm{P}<0.001 \mathrm{vs}$. control; ${ }^{\Delta \Delta} \mathrm{P}<0.01,{ }^{\Delta \Delta \Delta} \mathrm{P}<0.001 \mathrm{vs}$. I/R. HSP, heat shock protein; I/R, ischemia/reperfusion; ICA, icariin; NC, negative control.

A
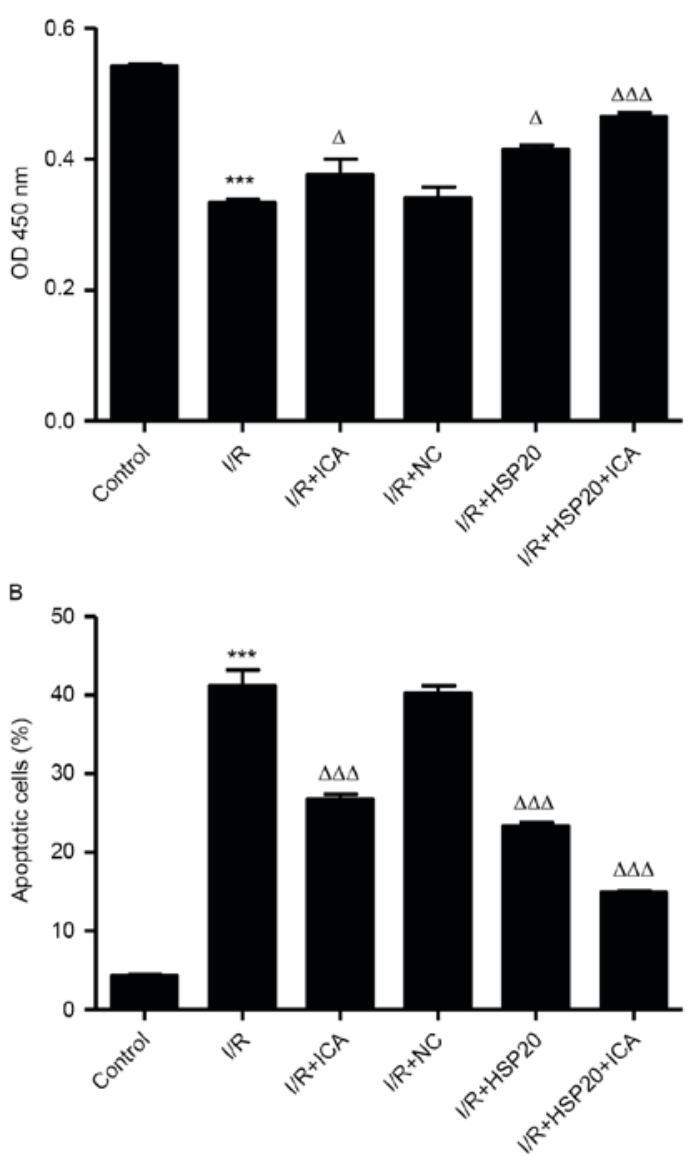

$\mathrm{C}$
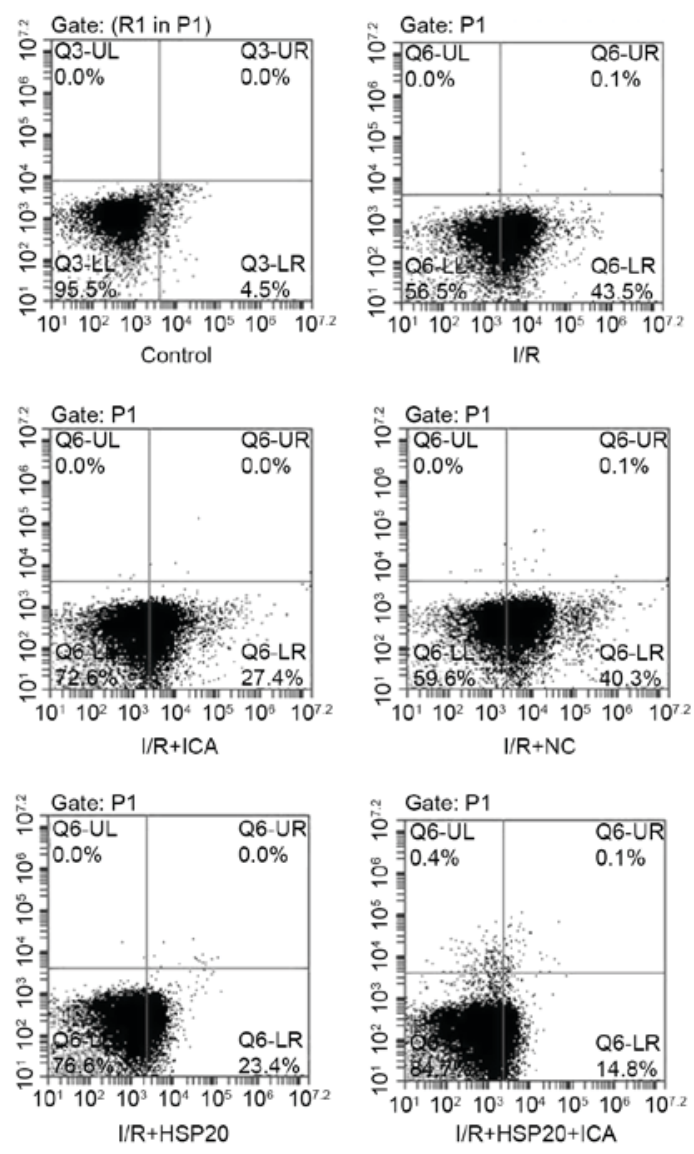

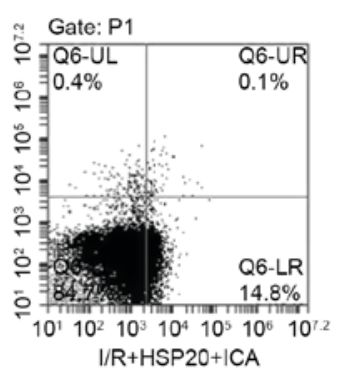

Figure 3. Effects of HSP20 overexpression on I/R-induced injury in H9C2 cells. (A) Cell proliferation and (B and C) apoptosis in H9C2 cells following I/R was evaluated by CCK-8 assay and flow cytometry. Data are presented as the mean \pm standard deviation. ${ }^{* * * *} \mathrm{P}<0.001$ vs. control; ${ }^{\Delta} \mathrm{P}<0.05,{ }^{\Delta \Delta \Delta} \mathrm{P}<0.001 \mathrm{vs}$. I/R HSP, heat shock protein; I/R, ischemia/reperfusion; NC, negative control; ICA, icariin. 
A

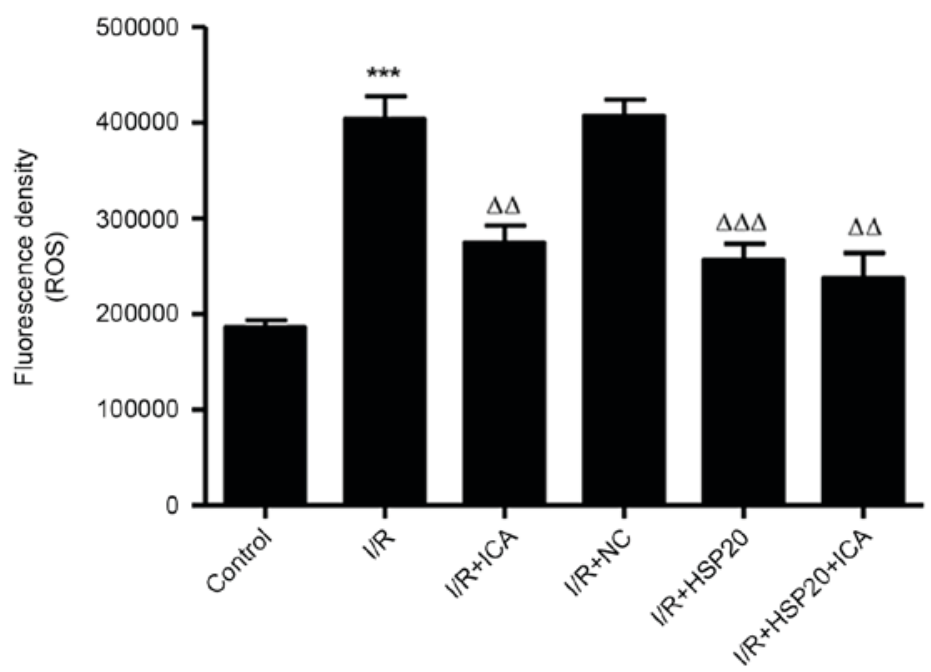

B

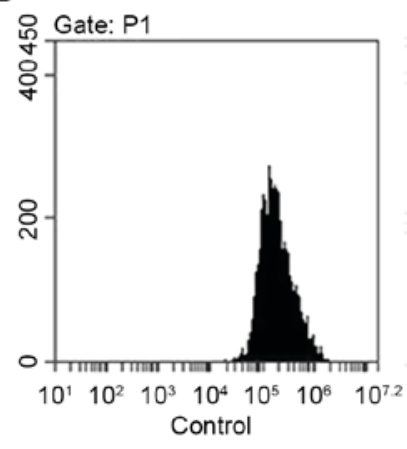

$\mathrm{E}$

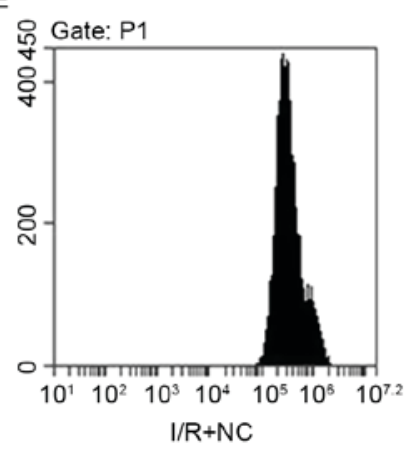

C

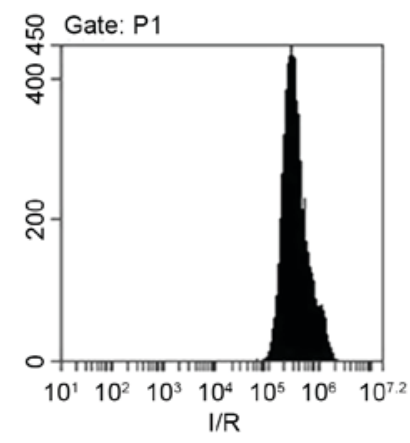

$\mathrm{F}$

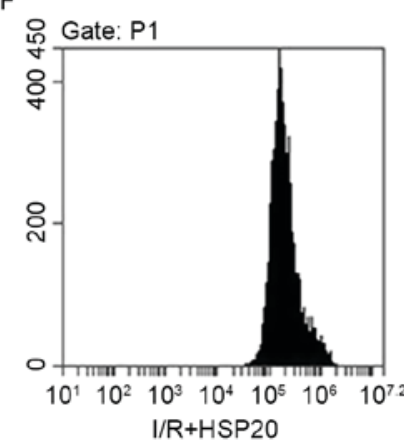

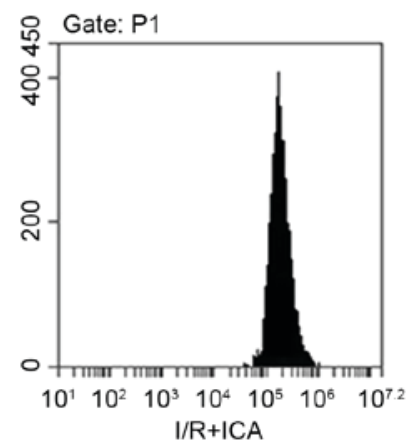

G

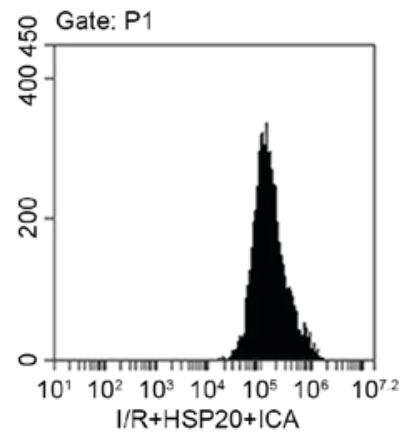

Figure 4. Effects of HSP20 overexpression on I/R-induced ROS production in H9C2 cells. (A) ROS production was evaluated by flow cytometry in H9C2 cells with different treatments, including (B) control, (C) I/R, (D) I/R+ICA, (E) I/R+NC, (F) I/R+HSP20 and (G) I/R+HSP20+ICA. Data are presented as the mean \pm standard deviation. ${ }^{* * * *} \mathrm{P}<0.001$ vs. control; ${ }^{\Delta \Delta} \mathrm{P}<0.01,{ }^{\Delta \Delta \Delta} \mathrm{P}<0.001$ vs. I/R. HSP, heat shock protein; I/R, ischemia/reperfusion; ROS, reactive oxygen species; NC, negative control; ICA, icariin.

I/R-induced H9C2 cells at them RNA and protein expression levels (Fig. 2C and D).

HSP20 overexpression promotes proliferation and inhibits $I / R$-induced apoptosis in H9C2 cells. The effects of I/R on $\mathrm{H} 9 \mathrm{C} 2$ cell proliferation and apoptosis were measured by CCK-8 and flow cytometry, respectively. As shown in Fig. 3A-C, I/R significantly inhibited cell proliferation and increased apoptosis in H9C2 cells compared with the control cells. Notably, HSP20 overexpression and/or ICA (10 $\mu \mathrm{mol} / \mathrm{l})$ treatment significantly increased cell proliferation and decreased apoptosis in H9C2 cells compared with the I/R group. These data indicate that ICA promotes cell proliferation and inhibits cell apoptosis of I/R-induced H9C2 cells by upregulating HSP20.
HSP20 overexpression inhibits I/R-induced ROS production in H9C2 cells. The effect of I/R on ROS production in H9C2 cells was measured by flow cytometry. As exhibited in Fig. 4, I/R significantly increased ROS production in H9C2 cells compared with the control cells. Notably, HSP20 overexpression and/or ICA $(10 \mu \mathrm{mol} / \mathrm{l})$ treatment significantly decreased ROS production in $\mathrm{H} 9 \mathrm{C} 2$ cells compared with the I/R group. These data demonstrate that ICA decreased ROS production in I/R-induced H9C2 cells by upregulating HSP20.

Western blot analysis evaluated the protective mechanisms related proteins in $I / R$-induced $H 9 C 2$ cells. The effects of $\mathrm{I} / \mathrm{R}$ on protein expression levels in $\mathrm{H} 9 \mathrm{C} 2$ cells were analyzed by western blotting. As presented in Fig. $5 \mathrm{~A}$ and $\mathrm{B}, \mathrm{I} / \mathrm{R}$ 


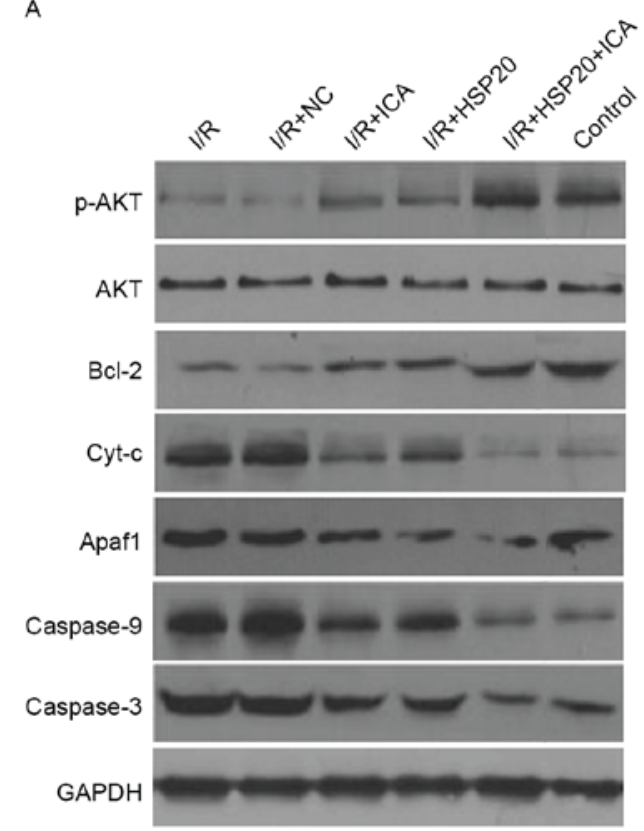

B

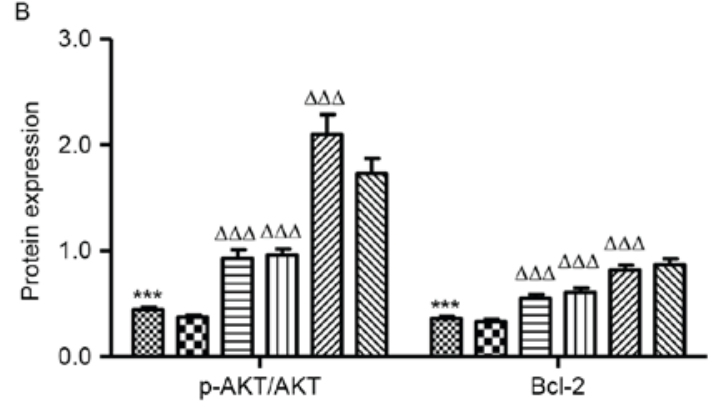

C

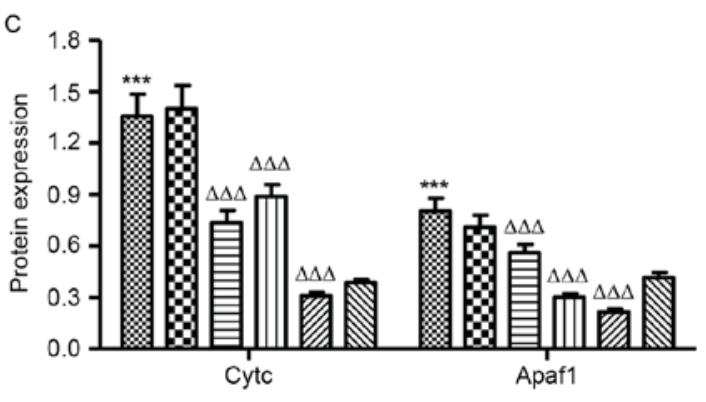

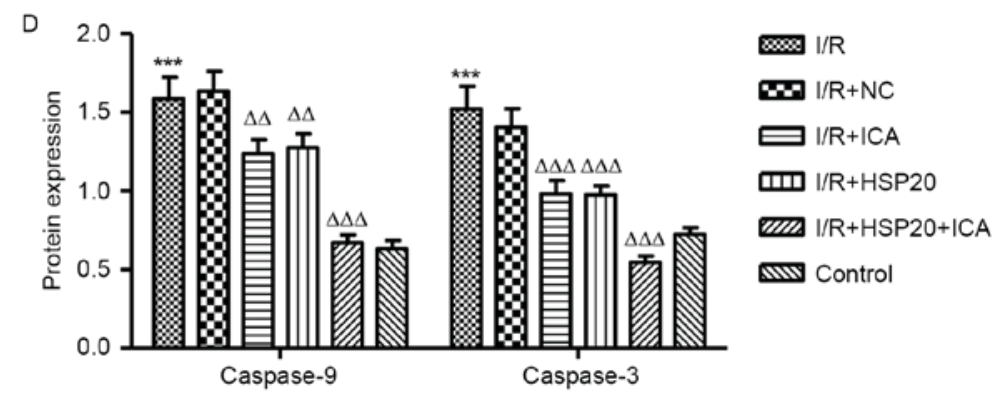

Figure 5. Effects of HSP20 overexpression on I/R-induced protein expression levels in H9C2 cells. Expression levels of p-Akt, Akt, Bcl-2, Cyt-c, APAF1, caspase- 9 and caspase- 3 in H9C2 cells following I/R treatment were analyzed by (A) western blotting and (B-D) quantified. Data are presented as the mean \pm standard deviation. ${ }^{* * *} \mathrm{P}<0.001$ vs. control; ${ }^{\Delta \Delta} \mathrm{P}<0.01,{ }^{\Delta \Delta \Delta} \mathrm{P}<0.001$ vs. I/R. HSP, heat shock protein; I/R, ischemia/reperfusion; $\mathrm{p}$, phosphorylated; Bcl-2, B-cell lymphoma 2; Cyt-c, cytochrome complex; APAF1, apoptotic protease activating factor 1; NC, negative control; ICA, icariin.

significantly decreased the expression level of p-AKT and Bcl-2 in $\mathrm{H} 9 \mathrm{C} 2$ cells when compared with the control cells. HSP20 overexpression and/or ICA $(10 \mu \mathrm{mol} / \mathrm{l})$ treatment significantly increased the expression level of p-AKT and Bcl-2 in the $\mathrm{H} 9 \mathrm{C} 2$ cells as compared with the I/R group. In addition, I/R significantly increased the expression levels of Cyt-c, APAF1, caspase- 9 and caspase- 3 in $\mathrm{H} 9 \mathrm{C} 2$ cells compared with the control (Fig. 5A, C and D). Notably, HSP20 overexpression and/or ICA $(10 \mu \mathrm{mol} / \mathrm{l})$ treatment significantly decreased the expression levels of Cyt-c, APAF1, caspase-9 and caspase-3 in $\mathrm{H} 9 \mathrm{C} 2$ cells compared with the I/R group. These data indicate that ICA inhibits apoptosis-associated protein expression levels in I/R-induced H9C2 cells by upregulating HSP20.

\section{Discussion}

Cardiomyocyte apoptosis maybe a fundamental aspect of the myocardial process that initiates or aggravates heart failure. Consistent with the previously reported cardioprotective effects of ICA $(14,15)$, it was found that ICA pretreatment promotes cardiomyocyte $\mathrm{H} 9 \mathrm{C} 2$ cell proliferation, and inhibits cell apoptosis and ROS production during the process of I/R injury. Other studies also demonstrated that ICA significantly attenuated cardiomyocyte apoptosis by downregulating Bcl-2/BCL2 associated $\mathrm{X}$, apoptosis regulator (Bax), matrix metallopeptidase (MMP)-2 and MMP-9 expression levels (27). It is well accepted that multiple genes are aberrantly expressed in infarct hearts, which are responsible for cardiac remodeling following $\mathrm{I} / \mathrm{R}$ (21). To the best of our knowledge, the present study is the first to demonstrate that ICA treatment protects against I/R-induced cardiomyocyte apoptosis and ROS production, which was associated with overexpression of HSP20 in vitro. These data demonstrate that HSP20 may exert a positive regulatory role in the treatment of I/R-induced cardiomyopathy.

ICA, the major active component isolated from Herbaepimedii, has been extensively investigated on protection against I/R injury and other stress stimuli; however, its possible protective effects on I/R-induced cardiotoxicity and underlying mechanisms are less well studied. Previously, Li et al (28) identified that anandamide enhanced HSP72 and HSP25 expression levels in the lungs to protect against lung inflammation, and acts as a cardioprotective against I/R insult via its induction of HSP72. The present study clearly demonstrates that overexpression of HSP20 significantly enhanced the protective effect of ICA on I/R-induced apoptosis and ROS production in $\mathrm{H} 9 \mathrm{C} 2$ cells. Furthermore, the 
apoptosis-associated markers, including Bcl-2, Cyt-c, APAF1, caspase- 9 and caspase- 3 were regulated by ICA treatment and HSP20 overexpression. Apoptosis is directly controlled by the Bcl-2 family, resulting in the translocation of Bax from the cytosol to the mitochondria and the release of Cyt-c (29), following the formation of the apoptosome together with APAF1 and caspase-9, which is followed by the activation of caspase-3 (30). HSP70 prevented cell apoptosis via associating with APAF1, as well as HSP27 that binds to Cyt-c and prevents Cyt-c-mediated interaction of APAF1 with caspase-9 (31). HSP60 and HSP10 expression in I/R-induced cardiomyocytes decreased the apoptotic cell number, Cyt-c release and caspase-3 activity (32). Consistent with the previous studies, the present data indicated that the expression levels of Cyt-c, APAF1, caspase- 9 and caspase- 3 were increased by I/R, but the level of $\mathrm{Bcl}-2$ expression was decreased. However, ICA treatment and HSP20 overexpression reduced the expression levels of Cyt-c, APAF1, caspase- 9 and caspase- 3 in $\mathrm{H} 9 \mathrm{C} 2$ cells induced by I/R. Indeed, the caspase-3 activity and percentage of myocardial apoptosis are increased upon I/R injury, but are decreased following ICA treatment (15). In addition, previous data demonstrated that lactate dehydrogenase release and caspase-3 activity in $\mathrm{H} 9 \mathrm{C} 2$ cells infected with recombinant adenovirus encoding wild-type HSP20 are also decreased (31). These results indicate that the protective effects of HSP20 are closely associated with mitochondrial function.

$\mathrm{PI} 3 \mathrm{~K} / \mathrm{Akt}$ is an intracellular signaling pathway, which is particularly important following ischemic insults. Activated Akt produces its anti-apoptotic effects via the phosphorylation of two categories of downstream substrates: The anti-apoptotic substrates $(\mathrm{Bcl}-2)$ and the pro-apoptotic substrates (caspase-9) $(7,33)$. Triptolide may be a potential neuroprotective agent for cerebral I/R injury associated with the activation of the PI3K/Akt/mechanistic target of rapamycin signaling pathway (34). ICA protects the heart against I/R injury and this protective effect of ICA may be associated with its anti-oxidative and anti-apoptotic actions involving the modulation of the PI3K-Akt signaling pathway (15). Furthermore, overexpression of HSP20 in the heart attenuates doxorubicin-induced cardiac injury, which appears to be dependent on Akt activation (35). In the present study, Akt inactivation was observed in I/R-induced H9C2 cells, which was inhibited by ICA treatment. Notably, HSP20 overexpression enhanced Akt activation in the $\mathrm{H} 9 \mathrm{C} 2$ cells induced by ICA treatment.

In conclusion, the present study provides the first evidence, to the best of our knowledge, that ICA treatment protects the heart against I/R-induced apoptosis and ROS production, and this protective effect of ICA may be associated with an associated upregulation of HSP20. Further research is required to confirm the cardioprotective effect of ICA on I/R and to clarify the molecular mechanisms involving the Akt signaling pathway using LY294002, a PI3K-Akt signaling pathway inhibitor. The current data indicate that HSP20 presents as a potential therapeutic protein for ischemic diseases and additional studies are necessary.

\section{References}

1. Donnan GA, Fisher M, Macleod M and Davis SM: Stroke. Lancet 371: 1612-1623, 2008.
2. Durukan A and Tatlisumak T: Acute ischemic stroke: Overview of major experimental rodent models, pathophysiology, and therapy of focal cerebral ischemia. Pharmacol Biochem Behav 87: 179-197, 2007.

3. Sanz-Rosa D, Garcia-Prieto J and Ibanez B: The future: Therapy of myocardial protection. Ann N Y Acad Sci 1254: 90-98, 2012.

4. Eltzschig HK and Eckle T: Ischemia and reperfusion-from mechanism to translation. Nat Med 17: 1391-1401, 2011.

5. Huang J, Upadhyay UM and Tamargo RJ: Inflammation in stroke and focal cerebral ischemia. Surg Neurol 66: 232-245, 2006.

6. Thind GS, Agrawal PR, Hirsh B, Saravolatz L, Chen-Scarabelli C, Narula $J$ and Scarabelli TM: Mechanisms of myocardial ischemia-reperfusion injury and the cytoprotective role of minocycline: Scope and limitations. Future Cardiol 11: 61-76, 2015.

7. Ke ZP, Xu P, Shi Y and Gao AM: MicroRNA-93 inhibits ischemia-reperfusion induced cardiomyocyte apoptosis by targeting PTEN. Oncotarget 7: 28796-28805, 2016.

8. Zhang F, Li ZL, Xu XM, Hu Y, Yao JH, Xu W, Jing HR, Wang S, Ning SL and Tian XF: Protective effects of icariin-mediated SIRT1/FOXO3 signaling pathway on intestinal ischemia/reperfusion-induced acute lung injury. Mol Med Rep 11: 269-276, 2015.

9. Wu J, Du J, Xu C, Le J, Liu B, Xu Y and Dong J: In vivo and in vitro anti-inflammatory effects of a novel derivative of icariin. Immunopharmacol Immunotoxicol 33: 49-54, 2011.

10. Li F, Gong QH, Wu Q, Lu YF and Shi JS: Icariin isolated from Epimedium brevicornum Maxim attenuates learning and memory deficits induced by d-galactose in rats. Pharmacol Biochem Behav 96: 301-305, 2010.

11. Tan HL, Chan KG, Pusparajah P, Saokaew S, Duangjai A, Lee LH and Goh BH: Anti-cancer properties of the naturally occurring aphrodisiacs: Icariin and its derivatives. Front Pharmacol 7: 191, 2016.

12. Chen YJ, Zheng HY, Huang XX, Han SX, Zhang DS, Ni JZ and He XY: Neuroprotective effects of icariin on brain metabolism, mitochondrial functions, and cognition in triple-transgenic Alzheimer's disease mice. CNS Neurosci Ther 22: 63-73, 2016.

13. Xiong D, Deng Y, Huang B, Yin C, Liu B, Shi J and Gong Q: Icariin attenuates cerebral ischemia-reperfusion injury through inhibition of inflammatory response mediated by NF- $x \mathrm{~B}, \mathrm{PPAR} \alpha$ and PPAR $\gamma$ in rats. Int Immunopharmacol 30: 157-162, 2016.

14. Meng X, Pei H and Lan C: Icariin exerts protective effect against myocardial Ischemia/Reperfusion injury in rats. Cell Biochem Biophys 73: 229-235, 2015.

15. Ke Z, Liu J, Xu P, Gao A, Wang L and Ji L: The cardioprotective effect of icariin on Ischemia-reperfusion injury in isolated rat heart: Potential involvement of the PI3K-Akt signaling pathway. Cardiovasc Ther 33: 134-140, 2015.

16. Strauch A and Haslbeck M: The function of small heat-shock proteins and their implication in proteostasis. Essays Biochem 60: 163-172, 2016.

17. Zoubeidi A and Gleave M: Small heat shock proteins in cancer therapy and prognosis. Int J Biochem Cell Biol 44: 1646-1656, 2012.

18. Muller P, Ruckova E, Halada P, Coates PJ, Hrstka R, Lane DP and Vojtesek B: C-terminal phosphorylation of Hsp70 and Hsp90 regulates alternate binding to co-chaperones CHIP and HOP to determine cellular protein folding/degradation balances Oncogene 32: 3101-3110, 2013.

19. Sahebkar A, Mohammadi A, Atabati A, Rahiman S, Tavallaie S, Iranshahi M, Akhlaghi S, Ferns GA and Ghayour-Mobarhan M: Curcuminoids modulate pro-oxidant-antioxidant balance but not the immune response to heat shock protein 27 and oxidized LDL in obese individuals. Phytother Res 27: 1883-1888, 2013.

20. Kennedy D, Jäger R, Mosser DD and Samali A: Regulation of apoptosis by heat shock proteins. IUBMB Life 66: 327-338, 2014.

21. De Celle T, Vanrobaeys F, Lijnen P, Blankesteijn WM, Heeneman S, Van Beeumen J, Devreese B, Smits JF and Janssen BJ: Alterations in mouse cardiac proteome after in vivo myocardial infarction: Permanent ischaemia versus ischaemia-reperfusion. Exp Physiol 90: 593-606, 2005.

22. Fan GC, Ren X, Qian J, Yuan Q, Nicolaou P, Wang Y, Jones WK, Chu G and Kranias EG: Novel cardioprotective role of a small heat-shock protein, Hsp20, against ischemia/reperfusion injury. Circulation 111: 1792-1799, 2005.

23. Fan GC, Yuan Q, Song G, Wang Y, Chen G, Qian J, Zhou X, Lee YJ, Ashraf M and Kranias EG: Small heat-shock protein Hsp20 attenuates beta-agonist-mediated cardiac remodeling through apoptosis signal-regulating kinase 1. Circ Res 99: 1233-1242, 2006. 
24. Ren XP, Wu J, Wang X, Sartor MA, Qian J, Jones K, Nicolaou P, Pritchard TJ and Fan GC: MicroRNA-320 is involved in the regulation of cardiac ischemia/reperfusion injury by targeting heat-shock protein 20. Circulation 119: 2357-2366, 2009.

25. Xiong S, Zheng Y, Jiang P, Liu R, Liu X and Chu Y: MicroRNA-7 inhibits the growth of human non-small cell lung cancer A549 cells through targeting BCL-2. Int J Biol Sci 7: 805-814, 2011.

26. Livak KJ and Schmittgen TD: Analysis of relative gene expression data using real-time quantitative PCR and the 2(-Delta Delta C(T)) method. Methods 25: 402-408, 2001.

27. Song YH, Li BS, Chen XM and Cai H: Ethanol extract from Epimedium brevicornum attenuates left ventricular dysfunction and cardiac remodeling through down-regulating matrix metalloproteinase- 2 and -9 activity and myocardial apoptosis in rats with congestive heart failure. Int J Mol Med 21: 117-124, 2008.

28. Li Q, Shi M and Li B: Anandamide enhances expression of heat shock protein 72 to protect against ischemia-reperfusion injury in rat heart. J Physiol Sci 63: 47-53, 2013.

29. Kuwana T, Mackey MR, Perkins G, Ellisman MH, Latterich M, Schneiter R, Green DR and Newmeyer DD: Bid, Bax, and lipids cooperate to form supramolecular openings in the outer mitochondrial membrane. Cell 111: 331-342, 2002.
30. Yao RQ, Qi DS, Yu HL, Liu J, Yang LH and Wu XX: Quercetin attenuates cell apoptosis in focal cerebral ischemia rat brain via activation of BDNF-TrkB-PI3K/Akt signaling pathway. Neurocheml Res 37: 2777-2786, 2012.

31. Zhu YH, Ma TM and Wang X: Gene transfer of heat-shock protein 20 protects against ischemia/reperfusion injury in rat hearts. Acta Pharmacol Sin 26: 1193-1200, 2005.

32. Lin KM, Lin B, Lian IY, Mestril R, Scheffler IE and Dillmann WH: Combined and individual mitochondrial HSP60 and HSP10 expression in cardiac myocytes protects mitochondrial function and prevents apoptotic cell deaths induced by simulated ischemia-reoxygenation. Circulation 103: 1787-1792, 2001.

33. Mocanu MM and Yellon DM: PTEN, the Achilles' heel of myocardial ischaemia/reperfusion injury? Br J Pharmacol 150: 833-838, 2007.

34. Li W, Yang Y, Hu Z, Ling S and Fang M: Neuroprotective effects of DAHP and Triptolide in focal cerebral ischemia via apoptosis inhibition and PI3K/Akt/mTOR pathway activation. Front Neuroanat 9: 48, 2015 .

35. Fan GC, Zhou X, Wang X, Song G, Qian J, Nicolaou P, Chen G, Ren X and Kranias EG: Heat shock protein 20 interacting with phosphorylated Akt reduces doxorubicin-triggered oxidative stress and cardiotoxicity. Circ Res 103: 1270-1279, 2008. 